\title{
Splitting Lemma for Biholomorphic Mappings with Smooth Dependence on Parameters
}

\section{Arkadiusz Lewandowski ${ }^{1}$}

Received: 21 April 2020 / Published online: 23 September 2020

(c) The Author(s) 2020

\section{Abstract}

We prove that the mappings obtained in Forstnerič splitting lemma vary in a $\mathcal{C}^{\left\lfloor\frac{l-1}{2}\right\rfloor}$ continuous way if only the input family of biholomorphic mappings close to Id (and their domains) is $\mathcal{C}^{l}$-continuous (see Theorem 1.3 for a precise formulation).

Keywords Strictly pseudoconvex domains · Forstnerič splitting lemma Cousin problems

Mathematics Subject Classification Primary 32H02 - Secondary 32T15

\section{Introduction}

Let $X$ be a complex manifold and let dist be a Riemannian distance function on $X$. For a set $U \subset X$ and a mapping $\gamma: U \rightarrow X$ put

$$
\operatorname{dist}_{U}(\gamma, \mathrm{Id}):=\sup _{x \in U} \operatorname{dist}(\gamma(x), x)
$$

where Id: $X \rightarrow X$ is an identity mapping. The so-called splitting lemma for biholomorphic maps close to identity due to Forstnerič reads as follows:

Theorem 1.1 Let $A, B \subset X$ be compact sets such that $A \cup B$ is a Stein compact and $\overline{A \backslash B} \cap \overline{B \backslash A}=\varnothing$. For an open set $C$ containing $A \cap B$, there exist open sets $A^{\prime}, B^{\prime}, C^{\prime}$ such that $A \subset A^{\prime}, B \subset B^{\prime}, A \cap B \subset C^{\prime} \subset A^{\prime} \cap B^{\prime} \subset C$, and constants $\varepsilon_{0}>0, c>0$, such that the following holds true: for any injective holomorphic

The author was supported by the Grant UMO-2017/26/D/ST1/00126 financed by the National Science Centre, Poland.

$凶$ Arkadiusz Lewandowski

Arkadiusz.Lewandowski@im.uj.edu.pl

1 Institute of Mathematics, Faculty of Mathematics and Computer Science, Jagiellonian University, Łojasiewicza 6, 30-348 Kraków, Poland 
mapping $\gamma: C \rightarrow X$ with $\operatorname{dist}_{C}(\gamma, \mathrm{Id})=: \varepsilon<\varepsilon_{0}$ there exist injective holomorphic mappings $\alpha: A^{\prime} \rightarrow X, \beta: B^{\prime} \rightarrow X$, depending continuously on $\gamma$, with

$$
\operatorname{dist}_{A^{\prime}}(\alpha, \mathrm{Id})<c \varepsilon, \quad \operatorname{dist}_{B^{\prime}}(\beta, \mathrm{Id})<c \varepsilon,
$$

and such that

$$
\gamma \circ \alpha=\beta \text { on } C^{\prime}
$$

If, moreover, $A \cap B$ is a Stein compact and $X^{\prime} \subset X$ is a closed complex subvariety such that $X^{\prime} \cap C=\varnothing$, then $\alpha$ and $\beta$ can be chosen tangent to the identity to any given finite order along $X^{\prime}$.

This is [4, Theorem 4.1] up to the type of the estimates (1.1), which are not necessarily linear in the quoted paper (the above formulation is [6, Theorem 9.7.1]). It is the crucial device in the construction of noncritical holomorphic functions and submersions from Stein manifolds ([4, Theorems 2.1 and 2.6]), or in the construction of the (families of the) exposing mappings of strictly pseudoconvex domains at boundary points (see $[2,3,13]$, etc.). It is worth mentioning that the first application of this latter type was made in [5] in the problem of constructing proper holomorphic embeddings of Riemann surfaces to $\mathbb{C}^{2}$.

From now on we restrict ourselves to the case where $X=\mathbb{C}^{n}$ and the distance is the Euclidean one, and to the situation where the interior of $A \cup B$ is a bounded strictly pseudoconvex domain. The latter three papers revealed the need for versions of splitting lemma with (smooth) parameters in the situation of this kind. Namely, it is desirable to know that if the mappings $\gamma_{t}$ and their domains of definition vary with certain regularity with parameter $t$, then the output mappings $\alpha_{t}$ and $\beta_{t}$ also behave decently with respect to parameters. [18, Theorem 1.1] says, roughly speaking, that if the mappings $\gamma_{t}$ together with their domains of definition vary in a continuous way with respect to parameter $t$ lying in a compact topological space, then so do the mappings $\alpha_{t}$ and $\beta_{t}$. We state it here for the convenience of the reader. Henceforth, for a set $S \subset \mathbb{C}^{n}$ and a real number $\mu$ we put $S(\mu):=\bigcup_{x \in S} \mathbb{B}(x, \mu)$, with $\mathbb{B}(x, \mu)$ standing for the Euclidean ball with center $x$ and radius $\mu$.

Theorem 1.2 ([18, Theorem 1.1]) Let $\mathcal{T}$ be a nonempty compact topological space, let $\left(A_{t}, B_{t}\right)_{t \in \mathcal{T}}$ be an admissible (in the sense of [18]) family of Cartan pairs in $\mathbb{C}^{n}$ (i.e., for all $t, A_{t}, B_{t}, A_{t} \cup B_{t}, A_{t} \cap B_{t}$ are Stein compacts such that $\left.\overline{A_{t} \backslash B_{t}} \cap \overline{B_{t} \backslash A_{t}}=\varnothing\right)$, and let $\mu>0$. Then there exists a $\tau>0$ such that for any $\eta>0$ there exists an $\varepsilon_{\eta}>0$ with the property that for any family $\left(\gamma_{t}:\left(A_{t} \cap B_{t}\right)(\mu) \rightarrow \mathbb{C}^{n}\right)_{t \in \mathcal{T}}$ of injective holomorphic mappings satisfying $\left\|\gamma_{t}-\mathrm{Id}\right\|_{\left(A_{t} \cap B_{t}\right)(\mu)}<\varepsilon_{\eta}, t \in \mathcal{T}$ and depending continuously with respect to all variables, there exist families $\left(\alpha_{t}: A_{t}(2 \tau) \rightarrow \mathbb{C}^{n}\right)_{t \in \mathcal{T}},\left(\beta_{t}: B_{t}(2 \tau) \rightarrow\right.$ $\left.\mathbb{C}^{n}\right)_{t \in \mathcal{T}}$ of injective holomorphic mappings, depending continuously on all variables, and such that for all $t \in \mathcal{T}$ we have

(1) $\gamma_{t}=\beta_{t} \circ \alpha_{t}^{-1}$ on $\left(A_{t} \cap B_{t}\right)(\tau)$, and

(2) $\left\|\alpha_{t}-\operatorname{Id}\right\|_{\left(A_{t}\right)(2 \tau)}<\eta,\left\|\beta_{t}-\operatorname{Id}\right\|_{\left(B_{t}\right)(2 \tau)}<\eta$. 
The most important case here seems to be the one where, for each $t, A_{t}$ is a small part of the closure of a strictly pseudoconvex domain $G_{t}$ around one of its boundary points and $B_{t}$ sums up with $A_{t}$ to give $\overline{G_{t}}$. In this context, we give a qualitative improvement of Theorem 1.2, where the input data depend in a regular way on parameters, and the output objects inherit some of this regularity - see Theorem 1.3 for the details. To give a precise statement, we first describe the situation we work within.

Let $M$ be a smooth second countable manifold and let $\left(G_{t}\right)_{t \in M}$ be a family of striclty pseudoconvex domains in $\mathbb{C}^{n}$ with boundaries of class $\mathcal{C}^{k}(k \geq 2)$, which vary in a $\mathcal{C}^{k}$-continuous way with respect to the parameter $t$ in the following sense: for each $t, G_{t}$ is given by a defining function $r_{t}$ such that $\nabla r_{t} \neq 0$ (nonvanishing gradient) and $\mathcal{L}_{r_{t}}>0$ (positive definite Levi form) on $U_{t}$, an open neighbourhood of $\partial G_{t}$, where for any fixed $t_{0} \in M, z_{0} \in U_{t_{0}}$, the function $(t, z) \mapsto r_{t}(z)$ is well-defined and of class $\mathcal{C}^{k}$ in a neighbourhood of $\left(t_{0}, z_{0}\right)$.

Let a smooth function $\varepsilon: M \rightarrow(0,1)$ such that for any $t \in M$ and any real number $\kappa$ with $|\kappa|<\varepsilon(t)$ the domain

$$
G_{t}^{(\kappa)}:=\left(G_{t} \backslash U_{t}\right) \cup\left\{z \in U_{t}: r_{t}(z)<\kappa\right\}
$$

is strictly pseudoconvex with boundary of class $\mathcal{C}^{k}$ and for a fixed $t \in M$ we have

$$
V_{t}:=\bigcup_{|\kappa|<\varepsilon(t)} \partial G_{t}^{(\kappa)} \subset \subset U_{t} .
$$

Note that, if the function $\varepsilon$ takes sufficiently small values, for each $t \in M$ and each $\zeta \in V_{t}$ there exists exactly one $\kappa=\kappa(t, \zeta)$ with $\zeta \in \partial G_{t}^{(\kappa)}$. Having this in mind, for $t \in M$ and $\zeta \in V_{t}$, let $A_{t, \zeta}, B_{t, \zeta} \subset \mathbb{C}^{n}$ be compact sets such that:

(i) $\varnothing \neq C_{t, \zeta}:=A_{t, \zeta} \cap B_{t, \zeta}$ is a Stein compact,

(ii) $A_{t, \zeta} \cup B_{t, \zeta}$ is the closure of its interior equal to $G_{t}^{(\kappa)}$, with the unique $\kappa$ such that $\zeta \in \partial G_{t}^{(\kappa)}$ as above, and $\zeta \in A_{t, \zeta} \backslash B_{t, \zeta}$,

(iii) $\overline{A_{t, \zeta} \backslash B_{t, \zeta}} \neq \varnothing, \overline{B_{t, \zeta} \backslash A_{t, \zeta}} \neq \varnothing$,

(iv) $\overline{A_{t, \zeta} \backslash B_{t, \zeta}} \cap \overline{B_{t, \zeta} \backslash A_{t, \zeta}}=\varnothing$,

(v) $\bigcup_{t \in M}\{t\} \times V_{t} \ni(t, \zeta) \mapsto \overline{A_{t, \zeta} \backslash B_{t, \zeta}}, \bigcup_{t \in M}\{t\} \times V_{t} \ni(t, \zeta) \mapsto \overline{B_{t, \zeta} \backslash A_{t, \zeta}}$, and $\bigcup_{t \in M}\{t\} \times V_{t} \ni(t, \zeta) \mapsto C_{t, \zeta}$ are continuous mappings to the space of nonempty compact subsets of $\mathbb{C}^{n}$, equipped with the topology induced by the Hausdorff distance,

(vi) there exists a smooth function $\mu: M \rightarrow(0,1)$ such that for all $t \in M, \zeta \in V_{t}$ and all $\sigma \in[0, \mu(t)]$ we have $A_{t, \zeta}(\sigma) \cap B_{t, \zeta}(\sigma)=C_{t, \zeta}(\sigma), \overline{A_{t, \zeta}(\sigma) \backslash B_{t, \zeta}(\sigma)} \cap$ $\overline{B_{t, \zeta}(\sigma) \backslash A_{t, \zeta}(\sigma)}=\varnothing$ (cf. [6, Lemma 5.7.4]), $G_{t}^{(\kappa)}(\sigma)$ is a strictly pseudoconvex domain with boundary of class $\mathcal{C}^{k}$, and $A_{t, \zeta}(\sigma), B_{t, \zeta}(\sigma), C_{t, \zeta}(\sigma)$ are the domains with boundaries piecewise of class $\mathcal{C}^{1}$.

The set of conditions (i)-(v) should be compared with the notion of admissibility of certain families of pairs of compact sets, from [18], already evoked in the formulation of Theorem 1.2.

Our main result now reads as follows. 
Theorem 1.3 Let $k, l, s \in \mathbb{N}, k \geq \max \{2, l+1\}$. For every family of pairs $\left(A_{t, \zeta}, B_{t, \zeta}\right)_{t \in M, \zeta \in V_{t}}$ of compact sets in $\mathbb{C}^{n}$, satisfying (i)-(vi) above, and for every smooth function $\mu: M \rightarrow(0,1)$ as in (vi), there exist smooth functions $\tau, \varepsilon_{0}: M \rightarrow$ $(0,1)$ and $C: M \rightarrow(0, \infty)$, with $2 \tau<\mu$, such that for any family of holomorphic injections

$$
\left(\gamma_{t, \zeta}: C_{t, \zeta}(\mu(t)) \rightarrow \mathbb{C}^{n}\right)_{t \in M, \zeta \in V_{t}}
$$

depending in a $\mathcal{C}^{l}$-continuous way on $(t, \zeta, z)$ (here $\gamma_{t, \zeta}$ is a function of variable $z$ ), and such that

$$
\sup _{x \in C_{t, \zeta}(\mu(t))}\left\|\gamma_{t, \zeta}(x)-x\right\|=: \varepsilon_{t, \zeta}<\varepsilon_{0}(t)
$$

there exist families of holomorphic injections

$$
\left(\alpha_{t, \zeta}: A_{t, \zeta}(2 \tau(t)) \rightarrow \mathbb{C}^{n}\right)_{t \in M, \zeta \in V_{t}}, \quad\left(\beta_{t, \zeta}: B_{t, \zeta}(2 \tau(t)) \rightarrow \mathbb{C}^{n}\right)_{t \in M, \zeta \in V_{t}},
$$

depending in a $\mathcal{C}^{\left\lfloor\frac{l-1}{2}\right\rfloor}$-continuous way on $(t, \zeta, z)$ and satisfying for all $t \in M$ and $\zeta \in V_{t}$ the following conditions:

(1) $\gamma_{t, \zeta} \circ \alpha_{t, \zeta}=\beta_{t, \zeta}$ on $C_{t, \zeta}(\tau(t))$,

(2) $\sup _{x \in A_{t, \zeta}(2 \tau(t))}\left\|\alpha_{t, \zeta}(x)-x\right\|, \sup _{x \in B_{t, \zeta}(2 \tau(t))}\left\|\beta_{t, \zeta}(x)-x\right\|<C(t) \varepsilon_{t, \zeta}$,

(3) $\alpha_{t, \zeta}$ interpolates identity to order $s$ at $\zeta$.

If we do not require the interpolation condition to be satisfied, in the construction above the Steinness of $C_{t, \zeta}$ is not needed.

Remark 1.4 The proof of Theorem 1.2 by Simon is based on the original proof of Theorem 1.1, given in [4]. This itself is by a rapidly convergent Kolmogorov-NashMoser type iteration scheme. There, the mappings $\alpha_{t}$ and $\beta_{t}$ are obtained as uniform limits of continuous mappings of all variables, which allows to deduce the continuity of these objects. This method seems not to be a proper one to use, once we are interested in smooth dependence on parameters. However, recently, in [6, Theorem 9.7.1] Forstneric gave a new proof of Theorem 1.1, relying on the implicit function theorem in Banach spaces. This is the method we use in the proof of Theorem 1.3. But yet, there emerge serious problems when trying to apply it to parametric case. The most challenging are:

(1) As observed in [18], there is, according to the author's knowledge, no parametric version of the implicit function theorem of wide enough generality to deduce that the mappings $\alpha_{t, \zeta}, \beta_{t, \zeta}$ depend in a regular way on parameters. In order to overcome this difficulty, we used the Whitney extension operators with parameters, which allowed us to translate the problem of the parameter dependence of those mappings to the situation solvable by using a nonparametric implicit function theorem (see Sect. 3 for the details).

(2) We need the parametric solution of the additive Cousin problem with bounds and fulfilling some parametric interpolation condition (see Lemma 1.8). Concerning 
the solution without the interpolation condition, we have constructed one in [13]. However, it had the drawback that the solution is on slightly smaller domains than the input data. This is not allowable here, because at the very end we need to use the implicit function theorem and therefore the partial derivatives $\frac{\partial \Theta_{t, \zeta}}{\partial c^{t, \zeta}}(\mathrm{Id}, 0)$ in (3.2) in Sect. 3 must be linear isomorphisms. Had we used methods of [13], they would only become injections. This requires a parametric solution of the $\bar{\partial}$-problem with extremely nice behaviour with respect to parameters. The one we need is given in [8, Theorem 4.10] (the space of parameters there is just $[0,1]$, but the analysis of the proof reveals that it can be adjusted to our situation).

Now, the interpolation condition with regular behaviour with respect to parameters is also nontrivial. In a nonparameter case, we deal with the following problem: with $f_{1}, \ldots, f_{m} \in \mathcal{O}\left(\mathbb{C}^{n}\right)$ given, that vanish to order $s$ at $\zeta$, and have no common zeros on $\bar{D}$, where $D$ is a strictly pseudoconvex domain, find holomorphic functions $g_{1}, \ldots, g_{m}$ in a neighbourhood of $\bar{D}$, such that

$$
\sum_{j=1}^{m} f_{j} g_{j}=1 .
$$

It is just the matter of the application of Oka-Cartan division theorem. However, this approach gives no information about the regularity with respect to parameters when all the objects are allowed to vary with some, and is therefore not applicable to our problem. Inspired by [14, pp. 324-326] and [12, p. 117] (see also [10]), we shall solve the above problem with parameters by constructing suitable family of Koszul complexes (see Sect. 2 for the details).

Remark 1.5 Note that the interpolation condition was also possible to be obtained in Theorem 1.2, as observed, for example, in [2].

Remark 1.6 The loss of regularity in Theorem 1.3 comes from two reasons: one is the use of (a variant of) in [8, Theorem 4.10]. Here, one would get somehow more precise result in terms of the families of functions of class $\mathcal{C}^{l, j}$ on the so called total space of the family of domains depending on a parameter, introduced in the quoted paper, but even in this language one needs to have $j \leq k-1$. This is just a technical improvement and therefore we decided to utilize the one-parameter language of functions of class $\mathcal{C}^{l}$ with respect to all variables. The second reason is that at the very end of the proof of Theorem 1.3, we need to interpret the family $\gamma_{t, \zeta}, \mathcal{C}^{l}$-continuous with respect to all variables, as a $\mathcal{C}^{m}$-continuous family of $\mathcal{C}^{m}$-continuous mappings on some compact set, for $(t, \zeta)$ close to a fixed parameter $\left(t_{0}, \zeta_{0}\right)$, with maximal possible $m$ (see Sect. 3 for the details).

We conjecture that the families $\alpha_{t, \zeta}, \beta_{t, \zeta}$ may be constructed to depend on a $\mathcal{C}^{l-1}$ continuous way with respect to all variables.

Remark 1.7 Some special case of Theorem 1.3 shall be one of the crucial devices in the construction of the $\mathcal{C}^{k}$-continuous family of exposing mappings of boundary points for the family of strictly pseudoconvex domains varying smoothly with the parameter, where the space of parameters is not necessarily compact (the paper in preparation); 
this would improve and extend the main result of [13], thus giving the comprehensive answer to the problem of the existence of such family, proposed in [1].

From now on, for an $m \in \mathbb{N}$ and an open set $S \subset \mathbb{C}^{n}$ with piecewise $\mathcal{C}^{1}$ boundary, we denote by $\mathcal{A}^{m}(S)$ the space of $\mathcal{C}^{m}$-continuous functions on $\bar{S}$ that are holomorphic in $S$. Similarly, we denote by $\mathcal{C}^{m}(\bar{S})$ the space of all $\mathcal{C}^{m}$-continuous functions on $\bar{S}$. We equip these spaces in standard norms turning them into Banach spaces. As indicated in Remark 1.4, we shall need the following parametric solution of the additive Cousin problem with bounds, which may be interesting on its own.

Lemma 1.8 Let $m \in \mathbb{N}$ and let the family of pairs of compact sets $\left(A_{t, \zeta}, B_{t, \zeta}\right)_{t \in M, \zeta \in V_{t}}$ as in Theorem 1.3. Then for sufficiently small smooth function $\tau: M \rightarrow(0,1)$ with $\tau<\mu$, where $\mu$ is as in Theorem 1.3, for all $t \in M$ and $\zeta \in V_{t}$ there exist bounded linear operators

$$
\begin{aligned}
& \mathfrak{A}_{t, \zeta}: \mathcal{A}^{m}\left(C_{t, \zeta}(\tau(t))\right) \rightarrow \mathcal{A}^{m}\left(A_{t, \zeta}(\tau(t))\right), \\
& \mathfrak{B}_{t, \zeta}: \mathcal{A}^{m}\left(C_{t, \zeta}(\tau(t))\right) \rightarrow \mathcal{A}^{m}\left(B_{t, \zeta}(\tau(t))\right)
\end{aligned}
$$

such that for all $t \in M, \zeta \in V_{t}$, and any $c^{t, \zeta} \in \mathcal{A}^{m}\left(C_{t, \zeta}(\tau(t))\right)$ we have

$$
c^{t, \zeta}=\mathfrak{B}_{t, \zeta}\left(c^{t, \zeta}\right)-\mathfrak{A}_{t, \zeta}\left(c^{t, \zeta}\right) \text { on } \overline{C_{t, \zeta}(\tau(t))} .
$$

The bounds of the operators $\mathfrak{A}_{t, \zeta}$ and $\mathfrak{B}_{t, \zeta}$ may be chosen to depend only on $t$, and in a smooth way.

Additionally, the operators $\mathfrak{A}_{t, \zeta}$ may be chosen in such a way that for any $c^{t, \zeta}$ the function $\mathfrak{A}_{t, \zeta}\left(c^{t, \zeta}\right)$ vanishes at $\zeta$ to order $s$.

Moreover, if the family $\left(c^{t, \zeta}\right)_{t \in M, \zeta \in V_{t}}$ depends in a $\mathcal{C}^{l}$-continuous way on all variables, then the families $\left(\mathfrak{A}_{t, \zeta}\left(c^{t, \zeta}\right)\right)_{t \in M, \zeta \in V_{t}}$ and $\left(\mathfrak{B}_{t, \zeta}\left(c^{t, \zeta}\right)\right)_{t \in M, \zeta \in V_{t}}$ depend in a $\mathcal{C}^{l-1}$ continuous way on all variables.

We close the introduction by recalling the notion of strictly pseudoconvex domains: a bounded domain $G \subset \mathbb{C}^{n}$ is called a strictly pseudoconvex one if there exist a neighborhood $U$ of $\partial G$ and a defining function $r: U \rightarrow \mathbb{R}$ of class $\mathcal{C}^{2}$ on $U$ and such that

(1) $G \cap U=\{z \in U: r(z)<0\}$,

(2) $\left(\mathbb{C}^{n} \backslash \bar{G}\right) \cap U=\{z \in U: r(z)>0\}$,

(3) $\operatorname{\nabla r}(z) \neq 0$ for $z \in \partial G$, where $\nabla r(z):=\left(\frac{\partial r}{\partial \bar{z}_{1}}(z), \cdots, \frac{\partial r}{\partial \bar{z}_{n}}(z)\right)$,

together with

$$
\mathcal{L}_{r}(z ; X)>0 \text { for } z \in \partial G \text { and nonzero } X \in T_{z}^{\mathbb{C}}(\partial G),
$$

where $\mathcal{L}_{r}$ denotes the Levi form of $r$ and $T_{z}^{\mathbb{C}}(\partial G)$ is the complex tangent space to $\partial G$ at $z$.

It is known that $U$ and $r$ can be chosen to satisfy (1)-(3) and, additionally:

(4) $\mathcal{L}_{r}(z ; X)>0$ for $z \in U$ and all nonzero $X \in \mathbb{C}^{n}$,

cf. [11].

We give the proof of Lemma 1.8 in Sect. 2. Theorem 1.3 is proved in Sect. 3. 


\section{Proof of Lemma 1.8}

Proof of Lemma 1.8 Let us start with some smooth function $\tau: M \rightarrow(0,1)$ with $\tau<\mu$. We shall decrease it appropriately during the proof.

Choose a family $\left(\chi_{t, \zeta}: \mathbb{C}^{n} \rightarrow[0,1]\right)_{t \in M, \zeta \in V_{t}}$ of smooth cutoff functions which depend in a smooth way on all variables and such that for all $t \in M, \zeta \in V_{t}$ we have $\chi_{t, \zeta}=0$ in a neighbourhood of $\overline{\overline{A_{t, \zeta}(\tau(t))} \backslash \overline{B_{t, \zeta}(\tau(t))}}, \chi_{t, \zeta}=1$ in a neighbourhood of $\overline{\overline{B_{t, \zeta}(\tau(t))} \backslash \overline{A_{t, \zeta}(\tau(t))}}$ and with spt $\chi_{t, \zeta}$ being some neighbourhood of this latter set. Observe that for a fixed $c^{t, \zeta} \in \mathcal{A}^{m}\left(C_{t, \zeta}(\tau(t))\right)$, the function $\chi_{t, \zeta} c^{t, \zeta}$ extends to a $\mathcal{C}^{m}$-continuous function on $\overline{A_{t, \zeta}(\tau(t))}$ vanishing on $\overline{\overline{A_{t, \zeta}(\tau(t))} \backslash \overline{B_{t, \zeta}(\tau(t))}}$. Similarly, $\left(\chi_{t, \zeta}-1\right) c^{t, \zeta}$ extends to a $\mathcal{C}^{m}$-continuous function on $\overline{B_{t, \zeta}(\tau(t))}$ vanishing on

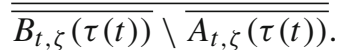

Given such a $c^{t, \zeta}$, consider the $(0,1)$-form

$$
\Lambda_{t, \zeta}:=c^{t, \zeta} \bar{\partial}_{z} \chi_{t, \zeta}
$$

with $\mathcal{C}^{m}$-continuous coefficients, defined on $A_{t, \zeta}(\tau(t)) \cup B_{t, \zeta}(\tau(t))=G_{t}^{(\kappa)}(\tau(t))$ and with support contained in $\overline{A_{t, \zeta}(\tau(t)) \cap B_{t, \zeta}(\tau(t))}$. Observe that in $A_{t, \zeta}(\tau(t)) \cup$ $B_{t, \zeta}(\tau(t))$ there is

$$
\Lambda_{t, \zeta}=\bar{\partial}_{z}\left(\chi_{t, \zeta} c^{t, \zeta}\right)=\bar{\partial}_{z}\left(\left(\chi_{t, \zeta}-1\right) c^{t, \zeta}\right)
$$

Let $g_{t, \zeta}^{j}(z):=\left(z^{j}-\zeta^{j}\right)^{s}, z \in \mathbb{C}^{n}, j=1, \ldots, n$, where for $w \in \mathbb{C}^{n}$ we have denoted by $w^{j}$ the $j$ th coordinate of $w$. All these functions are smooth with respect to all variables, vanish to order $s$ at $\zeta$, and

$$
\bigcap_{j=1}^{n}\left\{z \in \mathbb{C}^{n}: g_{t, \zeta}^{j}(z)=0\right\}=\{\zeta\} .
$$

Now we shall prepare to reaching the interpolation at $\zeta$. As indicated in Remark 1.4, for fixed $t$ and $\zeta$ it follows from Oka-Cartan division theorem that there exist functions $h_{t, \zeta}^{j}, j=1, \ldots, n$, holomorphic in a neighbourhood of $\overline{C_{t, \zeta}(\tau(t))}$ (if $\tau(t)$ is sufficiently small), and such that

$$
\sum_{j=1}^{n} h_{t, \zeta}^{j} g_{t, \zeta}^{j}=1
$$

in that neighbourhood. However, this approach gives no information about the regularity with respect to parameters. Therefore, we need to use a different method. Inspired by [14, pp. 324-326] and [12, p. 117] (see also [10]), we shall consider some family of suitably chosen Koszul complexes.

We first work with $t \in M$ and $\zeta \in V_{t}$ fixed. For such $t \in M, \zeta \in V_{t}$ let a positive constant $\tilde{\tau} \leq \tau(t)$ and a smoothly bounded strictly pseudoconvex domain 
$\widetilde{C}_{t, \zeta} \subset \mathbb{C}^{n} \backslash\{\zeta\}$ containing $\overline{C_{t, \zeta}(\widetilde{\tau})}$. Observe that there exist a positive constant $\delta$ such that

$$
\sum_{j=1}^{n}\left\|g_{t, \zeta}^{j}\right\|{\widetilde{\widetilde{C}_{t, \zeta}}} \geq \delta
$$

Take an open covering of $\widetilde{C}_{t, \zeta}$ constituted by

$$
U_{t, \zeta}^{j}:=\left\{z \in \widetilde{C}_{t, \zeta}:\left\|g_{t, \zeta}^{j}\right\|>\frac{\delta}{2 n}\right\}, \quad j=1, \ldots, n,
$$

and consider the smooth partition of unity $\left(\varphi_{t, \zeta}^{j}\right)_{j=1}^{n}$ subordinated to this covering. Define on $\widetilde{C}_{t, \zeta}$ the following functions

$$
\widetilde{h_{t, \zeta}^{j}}:=\frac{\varphi_{t, \zeta}^{j}}{g_{t, \zeta}^{j}}+\sum_{i=1}^{n} v_{t, \zeta}^{j i} g_{t, \zeta}^{j}, \quad j=1, \ldots, n,
$$

where the smooth functions $v_{t, \zeta}^{j i}$ satisfy $v_{t, \zeta}^{j i}=-v_{t, \zeta}^{i j}$ and are otherwise arbitrary for the moment. We want to find such functions that would force the holomorphicity of the functions $\widetilde{h_{t, \zeta}^{j}}$, with (2.2) being kept.

For still fixed $t \in M, \zeta \in V_{t}$ consider the following Koszul complex. Let $p, r, s \in$ $\mathbb{N}$ and let

$$
L_{r}^{t, \zeta}\left(\widetilde{C}_{t, \zeta}\right):=\left\{u \in \mathcal{C}_{0, r}^{p}\left(\widetilde{\widetilde{C}}_{t, \zeta}\right): \bar{\partial} u \in \mathcal{C}_{0, r+1}^{p}\left(\widetilde{\widetilde{C}}_{t, \zeta}\right)\right\}
$$

and

$$
L_{r}^{t, \zeta, s}\left(\widetilde{C}_{t, \zeta}\right):=L_{r}^{t, \zeta}\left(\widetilde{C}_{t, \zeta}\right) \otimes_{\mathbb{C}} \bigwedge^{s} \mathbb{C}^{n}
$$

that is, the space of linear combinations in $\bigwedge^{s} \mathbb{C}^{n}$ with coefficients from $L_{r}^{t, \zeta}\left(\widetilde{C}_{t, \zeta}\right)$. Let $e_{1}, \ldots, e_{n}$ be a canonical basis in $\mathbb{C}^{n}$ and let for $I=\left(i_{1}, \ldots, i_{q}\right)$ with $1 \leq i_{1}<$ $\ldots<i_{q} \leq n$

$$
e^{I}:=e_{i_{1}} \wedge \ldots \wedge e_{i_{q}}
$$

The elements of $L_{r}^{t, \zeta, s}\left(\widetilde{C}_{t, \zeta}\right)$ can be now represented as

$$
\sum_{|I|=q}^{\prime} u_{I} e^{I}
$$


where $\sum^{\prime}$ means summation over increasingly ordered sets $I$ and $u_{I} \in L_{r}^{t, \zeta}\left(\widetilde{C}_{t, \zeta}\right)$. We apply the operator $\bar{\partial}$ coefficientwise and in this convention

$$
\bar{\partial}: L_{r}^{t, \zeta, s}\left(\widetilde{C}_{t, \zeta}\right) \rightarrow L_{r+1}^{t, \zeta, s}\left(\widetilde{C}_{t, \zeta}\right)
$$

For $v=\sum_{|J|=w}^{\prime} v_{J} e^{J} \in L_{y}^{t, \zeta, w}\left(\widetilde{C}_{t, \zeta}\right)$ let

$$
u \wedge v:=\sum_{|I|=q,|J|=w}^{\prime}\left(u_{I} \wedge v_{J}\right) e^{I} \wedge e^{J} \in L_{r+y}^{t, \zeta, s+w}\left(\widetilde{C}_{t, \zeta}\right) .
$$

Further, if for $j \in I, j=i_{v}$ we put

$$
\left.e_{j}\right\lrcorner e^{I}:=(-1)^{\nu-1} e^{\left(i_{1}, \ldots, i_{v-1}, i_{v+1}, \ldots, i_{s}\right)},
$$

and $\left.e_{j}\right\lrcorner e^{I}=0$ for $j \notin I$, we may define a homological operator

$$
P_{1}: L_{r}^{t, \zeta, s}\left(\widetilde{C}_{t, \zeta}\right) \rightarrow L_{r}^{t, \zeta, s-1}\left(\widetilde{C}_{t, \zeta}\right)
$$

by

$$
\left.P_{1} u:=\sum_{j=1}^{N} \sum_{|I|=s}^{\prime}\left(g_{t, \zeta}^{j} u_{I}\right) e_{j}\right\lrcorner e^{I} .
$$

Then $P_{1}^{2}=0, P_{1}$ commutes with $\bar{\partial}$, and for $u \in L_{r}^{t, \zeta, s}\left(\widetilde{C}_{t, \zeta}\right), v \in L_{y}^{t, \zeta, w}\left(\widetilde{C}_{t, \zeta}\right)$ we have

$$
P_{1}(u \wedge v)=\left(P_{1} u\right) \wedge v+(-1)^{s} u \wedge\left(P_{1} v\right)
$$

and if we put

$$
\widetilde{h_{t, \zeta}}:=\sum_{j=1}^{n} \widetilde{h_{t, \zeta}^{j}} e_{j}
$$

then

$$
P_{1} \widetilde{h_{t, \zeta}}=1
$$

We need two auxiliary lemmata.

Lemma 2.1 For $u \in L_{r}^{t, \zeta, s}\left(\widetilde{C}_{t, \zeta}\right)$ with $P_{1} u=0$ there exists an $\omega \in L_{r}^{t, \zeta, s+1}\left(\widetilde{C}_{t, \zeta}\right)$ such that $P_{1} \omega=u$. 
Proof of Lemma 2.1 Define $\omega_{0}:=\sum_{j=1}^{n} \frac{\varphi_{t, \zeta}^{j}}{g_{t, \zeta}^{j}} \otimes e_{j} \in L_{0}^{t, \zeta, 1}\left(\widetilde{C}_{t, \zeta}\right)$. Then $P_{1} \omega_{0} \in$ $L_{0}^{0}\left(\widetilde{C}_{t, \zeta}\right)$ and $P_{1} \omega_{0}=1$. Therefore,

$$
\omega:=\omega_{0} \wedge u
$$

is what we are looking for.

Lemma 2.2 Let $u \in L_{r}^{t, \zeta, s}$ satisfy $P_{1} u=\bar{\partial} u=0$. Then there exist $\widehat{C_{t, \zeta}}$, a smoothly bounded strictly pseudoconvex domain with $\overline{C_{t, \zeta}(\tilde{\tau})} \subset \subset \widehat{C_{t, \zeta}} \subset \subset \widehat{C_{t, \zeta}, \widehat{C_{t, \zeta}}}$ arbitrarily close to $\widetilde{C_{t, \zeta}}$, and an $\omega \in L_{r}^{t, \zeta, s+1} \widehat{\left(C_{t, \zeta}\right)}$ such that $P_{1} \omega=u$ and $\bar{\partial} \omega=0$, both on $\widehat{C_{t, \zeta}}$.

Proof of Lemma 2.2 This is a (finite) descending induction with respect to $s+r$. If $\max \{s, r\}>n$ then the result trivially holds. Let the lemma hold true for bigger values of $s+r$. From Lemma 2.1 there exists an $\omega^{\prime} \in L_{r}^{t, \zeta, s+1}\left(\widetilde{C}_{t, \zeta}\right)$ with $P_{1} \omega^{\prime}=u$. Then, by inductive assumption, there exists an $\omega^{\prime \prime} \in L_{r+1}^{t, \zeta, s+2}\left(\widetilde{C_{t, \zeta}}\right)$ on some smoothly bounded strictly pseudoconvex domain $\overline{C_{t, \zeta}(\tilde{\tau})} \subset \subset \overline{C_{t, \zeta}} \subset \subset \overline{C_{t, \zeta}}, \overline{C_{t, \zeta}}$ arbitrarily close to $\widetilde{C_{t, \zeta}}$, such that $P_{1} \omega^{\prime \prime}=\bar{\partial} \omega^{\prime}$ and $\bar{\partial} \omega^{\prime \prime}=0$ on $\widetilde{C_{t, \zeta}}$. Let neighbourhoods

$$
\overline{C_{t, \zeta}(\tilde{\tau})} \subset \subset V_{0}^{t, \zeta} \subset \subset V^{t, \zeta} \subset \subset \overline{C_{t, \zeta}},
$$

arbitrarily close to $\widetilde{C_{t, \zeta}}$ (one may choose such because of strict pseudoconvexity of this latter set) and the solution operator for $\bar{\partial}$ problem

$$
T_{r}^{V^{t, \zeta}, V_{0}^{t, \zeta}}: \mathcal{C}_{0, r}\left(\overline{V^{t, \zeta}}\right) \rightarrow \mathcal{C}_{0, r-1}\left(V_{0}^{t, \zeta}\right)
$$

as in [17, Theorem V.2.5], and apply it coefficientwise. Thus there exists an $\omega^{\prime \prime \prime} \in$ $L_{r}^{t, \zeta, s+2} \widehat{\left(C_{t, \zeta}\right)}$, with smoothly bounded strictly pseudoconvex domain $\overline{C_{t, \zeta}(\widetilde{\tau})} \subset \subset$ $\widehat{C_{t, \zeta}} \subset \subset \widetilde{C_{t, \zeta}, \widehat{C_{t, \zeta}}}$ arbitrarily close to $\widetilde{C_{t, \zeta}}$, such that $\bar{\partial} \omega^{\prime \prime \prime}=\omega^{\prime \prime}$ on $\widehat{C_{t, \zeta}}$. Define

$$
\omega:=\omega^{\prime}-P_{1} \omega^{\prime \prime \prime}
$$

on $\widehat{C_{t, \zeta}}$, and this finishes the induction step.

Let us come back to the issue of the "improvement" of functions $\widetilde{h_{t, \zeta}^{j}}$. Obviously $\widetilde{\partial h_{t, \zeta}} \in L_{1}^{1}\left(\widetilde{C}_{t, \zeta}\right)$ (recall the definition of $\widetilde{h_{t, \zeta}}$ in (2.3)). Also, $P_{1} \widetilde{\partial h_{t, \zeta}}=0$ by (2.4).

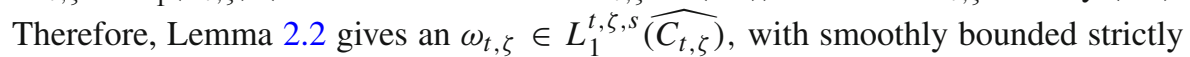
pseudoconvex domain $\overline{C_{t, \zeta}(\tilde{\tau})} \subset \subset \widehat{C_{t, \zeta}} \subset \subset \widetilde{C_{t, \zeta}, \widehat{C_{t, \zeta}}}$ arbitrarily close to $\widetilde{C_{t, \zeta}}$, such that $P_{1} \omega_{t, \zeta}=\widehat{\partial h_{t, \zeta}}$ and $\bar{\partial} \omega_{t, \zeta}=0$ on $\widehat{C_{t, \zeta}}$. Let open sets

$$
\overline{C_{t, \zeta}(\tilde{\tau})} \subset \subset{\widetilde{V_{0}}}^{t, \zeta} \subset \subset \widetilde{V}^{t, \zeta} \subset \subset \widetilde{C_{t, \zeta}},
$$


arbitrarily close to $\widetilde{C_{t, \zeta}}$, and the solution operator for the $\bar{\partial}$ problem

$$
T_{1}^{\widetilde{V}^{t, \zeta},{\widetilde{V_{0}}}^{t, \zeta}}: \mathcal{C}_{0,1}\left(\overline{\widetilde{V}^{t, \zeta}}\right) \rightarrow \mathcal{C}\left(\overline{\widetilde{V}_{0}^{t, \zeta}}\right)
$$

again as in [17, Theorem V.2.5]. Then

$$
k_{t, \zeta}:=T_{1}^{\widetilde{V}^{t, \zeta}, \widetilde{V}_{0}^{t, \zeta}} \omega_{t, \zeta}
$$

is in $L_{0}^{2}\left(K_{t, \zeta}\right)$ with some open set $\overline{C_{t, \zeta}(\widetilde{\tau})} \subset \subset K_{t, \zeta} \subset \subset \widetilde{C_{t, \zeta}}, K_{t, \zeta}$ arbitrarily close to $\widetilde{C_{t, \zeta}}$, and on that set $\bar{\partial} k_{t, \zeta}=\omega_{t, \zeta}$. Put

$$
h_{t, \zeta}:=\widetilde{h_{t, \zeta}}-P_{1} k_{t, \zeta} \in L_{0}^{1}\left(K_{t, \zeta}\right)
$$

Then $\bar{\partial} h_{t, \zeta}=0$ and $P_{1} h_{t, \zeta}=1$. Therefore, if we denote

$$
h_{t, \zeta}=: \sum_{j=1}^{n} h_{t, \zeta}^{j} e_{j}
$$

then the functions $h_{t, \zeta}^{j}$ are what we were after (for $t$ and $\zeta$ fixed).

Now, note that by the construction, the functions

$$
(s, \xi, w) \mapsto h_{s, \xi}^{1}(w), \ldots,(s, \xi, w) \mapsto h_{s, \xi}^{n}(w)
$$

are $\mathcal{C}^{l}$-continuous near a fixed $(t, \zeta, z)$. Finally, suitable partition of unity argument gives the $\mathcal{C}^{l}$-continuous families of holomorphic functions

$$
\left(h_{t, \zeta}^{1}\right)_{t \in M, \zeta \in V_{t}}, \ldots,\left(h_{t, \zeta}^{n}\right)_{t \in M, \zeta \in V_{t}}
$$

in neighbourhoods of sets $\overline{C_{t, \zeta}(\tau(t))}$ (after possible decreasing of the function $\tau$ ), such that (2.1) holds.

Now we are ready to reach the interpolation condition and so we continue with the proof of Lemma 1.8. After possible again decreasing the function $\tau$, for $c^{t, \zeta} \in$ $\mathcal{A}^{m}\left(C_{t, \zeta}(\tau(t))\right)$ let us define

$$
\begin{aligned}
& \mathfrak{A}_{t, \zeta}\left(c^{t, \zeta}\right)(z):=-\chi_{t, \zeta}(z) c^{t, \zeta}(z)+\sum_{j=1}^{n} g_{t, \zeta}^{j}(z) S_{t, \zeta}\left(h_{t, \zeta}^{j} \Lambda_{t, \zeta}\right)(z), \quad z \in \overline{A_{t, \zeta}(\tau(t))}, \\
& \mathfrak{B}_{t, \zeta}\left(c^{t, \zeta}\right)(z):=-\left(\chi_{t, \zeta}(z)-1\right) c^{t, \zeta}(z) \\
& \quad+\sum_{j=1}^{n} g_{t, \zeta}^{j}(z) S_{t, \zeta}\left(h_{t, \zeta}^{j} \Lambda_{t, \zeta}\right)(z), \quad z \in \overline{B_{t, \zeta}(\tau(t))},
\end{aligned}
$$


where $S_{t, \zeta}$ is the solution operator for the $\bar{\partial}$ problem on $G_{t}^{(\kappa)}(\tau(t))$, as given by [8, Theorem 4.10] (see also [15], which is utilized there); see (2) in Remark 1.4. By a similar argument as the one given in the quoted paper, we may show that the dependence of $S_{t, \zeta}\left(h_{t, \zeta}^{j} \Lambda_{t, \zeta}\right)(z)$ on all variables is of class $\mathcal{C}^{l-1}$, if only for $c^{t, \zeta}(z)$ it is of class $\mathcal{C}^{l}$. Observe that these objects satisfy the required properties. This finishes the proof.

\section{Proof of Theorem 1.3}

Proof of Theorem 1.3 Let $m=\left\lfloor\frac{l-1}{2}\right\rfloor$. Fix a smooth function $0<\tau<\frac{\mu}{2}$ on $M$, such that Lemma 1.8 holds with $2 \tau$. It suffices to find the families

$$
\left(\alpha_{t, \zeta} \in \mathcal{A}^{m}\left(A_{t, \zeta}(2 \tau(t))\right)\right)_{t \in M, \zeta \in V_{t}}, \quad\left(\beta_{t, \zeta} \in \mathcal{A}^{m}\left(B_{t, \zeta}(2 \tau(t))\right)\right)_{t \in M, \zeta \in V_{t}}
$$

of mappings depending in a $\mathcal{C}^{m}$-continuous way on all variables and such that (1)-(3) of Theorem 1.3 hold true. Then, in virtue of [18, Lemma 4.2] (see also in [9, Lemma 2.1.3]) all the mappings $\alpha_{t, \zeta}$ and $\beta_{t, \zeta}$ will automatically be injective after possible slight decreasing of functions $\tau$ and $\varepsilon_{0}$.

Let $\Phi(x, z):=x+z, x, z \in \mathbb{C}^{n}$. Then for any $x \in \mathbb{C}^{n}$ the mapping $\Phi(x, \cdot)$ is a composition of shears and therefore a holomorphic automorphism of $\mathbb{C}^{n}$, such that its differential at $z=0$ equals Id. We have the following

Observation 1. For any complex manifold $S$ and any couple of mappings $f, g: S \rightarrow$ $\mathbb{C}^{n}$ there exists a unique mapping $c: S \rightarrow \mathbb{C}^{n}$ with $\Phi(f(s), c(s))=g(s), s \in S$, i.e. $c(s):=g(s)-f(s), s \in S$. Note that if $f$ and $g$ are holomorphic or continuous, then so is $c .^{1}$

Lemma 1.8 applied for $2 \tau$ gives us the possibility to define, for $c^{t, \zeta} \in$ $\mathcal{A}^{m}\left(C_{t, \zeta}(2 \tau(t))\right)$,

$$
\alpha_{c^{t, \zeta}}(z):=\Phi\left(z, \mathfrak{A}_{t, \zeta}\left(c^{t, \zeta}\right)(z)\right)=z+\mathfrak{A}_{t, \zeta}\left(c^{t, \zeta}\right)(z), \quad z \in A_{t, \zeta}(2 \tau(t)),
$$

and

$$
\beta_{c^{t, \zeta}}(z):=\Phi\left(z, \mathfrak{B}_{t, \zeta}\left(c^{t, \zeta}\right)(z)\right)=z+\mathfrak{B}_{t, \zeta}\left(c^{t, \zeta}\right)(z), \quad z \in B_{t, \zeta}(2 \tau(t)) .
$$

Observe that if $c^{t, \zeta}$ is close to $0_{t, \zeta}$, which from now on will denote the zero mapping restricted to $C_{t, \zeta}(2 \tau(t))$, then $\alpha_{c^{t, \zeta}}$ and $\beta_{c^{t, \zeta}}$ are close to Id restricted to $A_{t, \zeta}(2 \tau(t))$ and, respectively, to $B_{t, \zeta}(2 \tau(t))$. From now on, we shall denote by $\operatorname{Id}_{t, \zeta}$ the appropriate restrictions of the identity mapping (the domains of the restrictions should be clear from the context).

Choose the family $\left(C_{t, \zeta}^{0}\right)_{t \in M, \zeta \in V_{t}}$ of compact sets, being the closures of smoothly bounded domains, depending continuously on $t$ and $\zeta$, and such that

$$
C_{t, \zeta}(2 \tau(t)) \subset \subset \operatorname{int} C_{t, \zeta}^{0} \subset \subset C_{t, \zeta}(\mu(t)) .
$$

\footnotetext{
1 This is a trivial version of [6, Lemma 9.7.2].
} 
Observe that if $\gamma^{t, \zeta} \in \mathcal{A}^{m}\left(C_{t, \zeta}^{0}\right)$ is close to $\operatorname{Id}_{t, \zeta}$ on $C_{t, \zeta}^{0}$, then $\gamma^{t, \zeta} \circ \alpha_{c^{t, \zeta}}$ : $\overline{C_{t, \zeta}(2 \tau(t))} \rightarrow \mathbb{C}^{n}$ is a mapping from $\mathcal{A}^{m}\left(C_{t, \zeta}(2 \tau(t))\right)$, close to $\operatorname{Id}_{t, \zeta}$, if only $c^{t, \zeta} \in \mathcal{A}^{m}\left(C_{t, \zeta}(2 \tau(t))\right)$ is close to $0_{t, \zeta}$. Note also that $\alpha_{0_{t, \zeta}}=\operatorname{Id}_{t, \zeta}$ and $\beta_{0_{t, \zeta}}=$ $\operatorname{Id}_{t, \zeta}$.

Observation 1 gives, for fixed $t, \zeta$, a unique mapping $\tilde{c}^{t, \zeta} \in \mathcal{A}^{m}\left(C_{t, \zeta}(2 \tau(t))\right)$ such that

$$
\Phi\left(\gamma^{t, \zeta} \circ \alpha_{c^{t, \zeta}}(z), \tilde{c}^{t, \zeta}(z)\right)=\beta_{c^{t, \zeta}}(z), \quad z \in \overline{C_{t, \zeta}(2 \tau(t))},
$$

with $\mathcal{C}^{l-1}$-continuous dependence on all variables, if only the functions $\gamma^{t, \zeta}, c^{t, \zeta}$ are chosen so that they depend in a $\mathcal{C}^{l}$-continuous way on all variables.

To summarize, for fixed $t, \zeta$ we have the following equality

$$
\tilde{c}^{t, \zeta}=\beta_{c^{t, \zeta}}-\gamma^{t, \zeta} \circ \alpha_{c^{t, \zeta}} \text { on } \overline{C_{t, \zeta}(2 \tau(t))},
$$

where $\gamma^{t, \zeta}, c^{t, \zeta}$ are the input data. This means that we have defined, for $t, \zeta$ fixed, the mapping

$$
\Theta_{t, \zeta}\left(\gamma^{t, \zeta}, c^{t, \zeta}\right):=\tilde{c}^{t, \zeta}
$$

from a neighbourhood of $\left(\operatorname{Id}_{t, \zeta}, 0_{t, \zeta}\right)$ in $\mathcal{A}^{m}\left(C_{t, \zeta}^{0}\right) \times \mathcal{A}^{m}\left(C_{t, \zeta}(2 \tau(t))\right)$ into $\mathcal{A}^{m}\left(C_{t, \zeta}\right.$ $(2 \tau(t)))$, such that $\Theta_{t, \zeta}\left(\operatorname{Id}_{t, \zeta}, 0_{t, \zeta}\right)=0_{t, \zeta}$. Note that the size of the domain of definition of $\Theta_{t, \zeta}$ may be chosen to depend in a smooth way on $t, \zeta$.

Directly from the construction it follows that the mapping $\Theta_{t, \zeta}$ is smooth. Furthermore, it can be easily calculated that

$$
\frac{\partial \Theta_{t, \zeta}}{\partial c^{t, \zeta}}\left(\operatorname{Id}_{t, \zeta}, 0_{t, \zeta}\right)=\operatorname{Id}_{t, \zeta}
$$

Therefore, for fixed $t, \zeta$, the implicit mapping theorem guarantees that the equation

$$
\Theta_{t, \zeta}\left(\gamma^{t, \zeta}, c^{t, \zeta}\right)=0_{t, \zeta}
$$

has a unique local solution $c^{t, \zeta}=\psi_{t, \zeta}\left(\gamma^{t, \zeta}\right) \in \mathcal{A}^{m}\left(C_{t, \zeta}(2 \tau(t))\right)$ for any $\gamma^{t, \zeta} \in$ $\mathcal{A}^{m}\left(C_{t, \zeta}^{0}\right)$ close to $\operatorname{Id}_{t, \zeta}$, such that $\psi_{t, \zeta}\left(\operatorname{Id}_{t, \zeta}\right)=0_{t, \zeta}$.

Define

$$
\begin{aligned}
\alpha_{t, \zeta} & :=\alpha_{\psi_{t, \zeta}\left(\gamma_{t, \zeta}\right)}, \\
\beta_{t, \zeta} & :=\beta_{\psi_{t, \zeta}\left(\gamma_{t, \zeta}\right)} .
\end{aligned}
$$

Note the difference between the family of functions $\gamma_{t, \zeta}$ appearing in the assumption of the Theorem and the family of functions $\gamma^{t, \zeta}$ introduced in the construction of mapping $\Theta_{t, \zeta}: \gamma_{t, \zeta}$ is the special case of $\gamma^{t, \zeta}$, depending in a $\mathcal{C}^{l}$-continuous way on all variables. These mappings $\alpha_{t, \zeta}, \beta_{t, \zeta}$ are what we are looking for, provided the following holds true:

Claim. The mappings $\psi_{t, \zeta}\left(\gamma_{t, \zeta}\right)$ depend in a $\mathcal{C}^{m}$-continuous way on all variables. 
Proof of Claim Fix $t_{0}$ and $\zeta_{0}$. For $(s, \xi)$ sufficiently close to $\left(t_{0}, \zeta_{0}\right)$, say from an open neighbourhood $U_{t_{0}, \zeta_{0}}$, choose compact sets $C^{0}, K, K^{\prime} \subset \mathbb{C}^{n}$ such that

$$
\begin{gathered}
\bigcup_{(s, \xi) \in U_{t_{0}, \zeta_{0}}} C_{s, \xi}(2 \tau(s)) \subset C^{0} \subset \bigcap_{(s, \xi) \in U_{t_{0}, \zeta_{0}}} C_{s, \xi}^{0}, \\
\bigcup_{(s, \xi) \in U_{t_{0}, \zeta_{0}}} \overline{A_{s, \xi}(2 \tau(s)) \cup B_{s, \xi}(2 \tau(s))} \subset K, \\
\bigcup_{(s, \xi) \in U_{t_{0}, \zeta_{0}}} C_{s, \xi}(2 \tau(s)) \subset \subset K^{\prime} \subset \subset K \cap C^{0},
\end{gathered}
$$

and $K^{\prime}, K$ are the closures of some smoothly bounded domains. We shall consider some modifications of the operators $\mathfrak{A}_{s, \xi}, \mathfrak{B}_{s, \xi}$ from Lemma 1.8. Namely, for a $c \in$ $\mathcal{C}^{m}\left(K^{\prime}\right), z \in K^{\prime}$, and $(s, \xi) \in U_{t_{0}, \zeta_{0}}$ (after possible shrinking $U_{t_{0}, \zeta_{0}}$ ) we define

$$
\begin{aligned}
& \widetilde{\mathfrak{A}}_{s, \xi}(c)(z):=-\chi_{s, \xi}(z) c(z)+\Upsilon_{s, \xi}\left(\sum_{j=1}^{n} g_{s, \xi}^{j} S_{s, \xi}\left(h_{s, \xi}^{j} \Lambda_{s, \xi}\right)\right)(z), \\
& \widetilde{\mathfrak{B}}_{s, \xi}(c)(z):=-\left(\chi_{s, \xi}(z)-1\right) c(z)+\Upsilon_{s, \xi}\left(\sum_{j=1}^{n} g_{s, \xi}^{j} S_{s, \xi}\left(h_{s, \xi}^{j} \Lambda_{s, \xi}\right)\right)(z),
\end{aligned}
$$

where

$$
\Upsilon_{s, \xi}: \mathcal{C}^{m}\left(\overline{A_{s, \xi}(2 \tau(s)) \cup B_{s, \xi}(2 \tau(s))}\right) \rightarrow \mathcal{C}^{m}\left(\mathbb{C}^{n}\right)
$$

is the Whitney extension (linear) operator ([19]). Due to the work of Glaeser ([7]), there exists a constant $D$ such that for $s, \xi$ close to $t_{0}, \zeta_{0}$ we have

$$
\left\|\Upsilon_{s, \xi}(f)\right\|_{m, K} \leq D\|f\|_{m, \overline{A_{s, \xi}(2 \tau(s)) \cup B_{s, \xi}(2 \tau(s))}}, \quad f \in \mathcal{C}^{m}\left(\overline{A_{s, \xi}(2 \tau(s)) \cup B_{s, \xi}(2 \tau(s))}\right) .
$$

Further, for a $c \in \mathcal{C}^{m}\left(K^{\prime}\right), z \in K^{\prime}$, and $s, \xi$ close to $t_{0}, \zeta_{0}$ let

$$
\begin{aligned}
& \widetilde{\alpha_{c}^{s, \xi}}(z):=z+\widetilde{\mathfrak{A}}_{s, \xi}(c)(z), \\
& \widetilde{\beta_{c}^{s, \xi}}(z):=z+\widetilde{\mathfrak{B}}_{s, \xi}(c)(z),
\end{aligned}
$$

and finally, for $(\gamma, c) \in \Omega \subset \mathcal{A}^{m}\left(C^{0}\right) \times \mathcal{C}^{m}\left(K^{\prime}\right)$, where $\Omega$ is an open neighbourhood of (Id, 0$)$, and for $(s, \xi) \in U_{t_{0}, \zeta_{0}}$, after possible again shrinking of $U_{t_{0}, \zeta_{0}}$, we may define the mapping

$$
\Theta: U \times \Omega \ni(s, \xi, \gamma, c) \mapsto \tilde{c}:=\widetilde{\beta_{c}^{s, \xi}}-\gamma \circ \widetilde{\alpha_{c}^{s, \xi}} \in \mathcal{C}^{m}\left(K^{\prime}\right)
$$

Now, an inspection of the proof of Whitney extension theorem (with additions due to Glaeser) given in [16, Theorem 3.2 and Complement 3.5], allows us to deduce that $\Theta$ 
is of class $\mathcal{C}^{m}$. Moreover, $\Theta\left(t_{0}, \zeta_{0}, \mathrm{Id}, 0\right)=0$, and it may be calculated that

$$
\frac{\partial \Theta}{\partial c}\left(t_{0}, \zeta_{0}, \mathrm{Id}, 0\right)=\mathrm{Id}
$$

The implicit mapping theorem yields that the equation

$$
\Theta(s, \xi, \gamma, c)=0
$$

has a unique local solution

$$
c=\psi(s, \xi, \gamma) \in \mathcal{C}^{m}\left(K^{\prime}\right)
$$

for $s, \xi$ close to $t_{0}, \zeta_{0}$ and $\gamma$ close to Id, with $\psi\left(t_{0}, \zeta_{0}\right.$, Id $)=0$.

Notice now that the mappings $\Theta_{t, \zeta}$ above (see (3.1)) could have been as well constructed for the functions $c^{t, \zeta}$ from $\mathcal{C}^{m}\left(\overline{C_{t, \zeta}(2 \tau(t))}\right)$ instead of $\mathcal{A}^{m}\left(C_{t, \zeta}(2 \tau(t))\right.$, and we could have used the implicit mapping theorem to find the unique local solutions of the equations

$$
\Theta_{t, \zeta}\left(\gamma^{t, \zeta}, c^{t, \zeta}\right)=0_{t, \zeta}
$$

say

$$
\widetilde{c^{t, \zeta}}=\widetilde{\psi_{t, \zeta}}\left(\gamma^{t, \zeta}\right) \in \mathcal{C}^{m}\left(\overline{C_{t, \zeta}(2 \tau(t))}\right)
$$

for any $\gamma^{t, \zeta} \in \mathcal{A}^{m}\left(C_{t, \zeta}^{0}\right)$ close to $\operatorname{Id}_{t, \zeta}$, such that $\widetilde{\psi_{t, \zeta}}\left(\operatorname{Id}_{t, \zeta}\right)=0_{t, \zeta}$. But then, for $\gamma^{t, \zeta}$ sufficiently close to $\operatorname{Id}_{t, \zeta}$, it must be equal to $\psi_{t, \zeta}\left(\gamma^{t, \zeta}\right) \in \mathcal{A}^{m}\left(C_{t, \zeta}(2 \tau(t))\right)$. Finally, observe that $\psi(s, \xi, \gamma)$ restricted to $\overline{C_{s, \xi}(2 \tau(s))}$ solves

$$
\Theta_{s, \xi}(\gamma, c)=0_{s, \xi}
$$

in $\mathcal{C}^{m}\left(\overline{C_{s, \xi}(2 \tau(s))}\right)$. In particular, $\psi_{t, \zeta}\left(\gamma_{t, \zeta}\right)$ depend in a $\mathcal{C}^{m}$-continuous way on all variables. This concludes the proof of the Claim.

Now $\alpha_{t, \zeta}, \beta_{t, \zeta}$ are exactly what we were after and the Theorem is proved.

Acknowledgements The author would like to thank the referee for a very careful reading and valuable remarks, which helped to greatly improve the paper.

Open Access This article is licensed under a Creative Commons Attribution 4.0 International License, which permits use, sharing, adaptation, distribution and reproduction in any medium or format, as long as you give appropriate credit to the original author(s) and the source, provide a link to the Creative Commons licence, and indicate if changes were made. The images or other third party material in this article are included in the article's Creative Commons licence, unless indicated otherwise in a credit line to the material. If material is not included in the article's Creative Commons licence and your intended use is not permitted by statutory regulation or exceeds the permitted use, you will need to obtain permission directly from the copyright holder. To view a copy of this licence, visit http://creativecommons.org/licenses/by/4.0/. 


\section{References}

1. Deng, F., Guan, Q., Zhang, L.: Properties of squeezing functions and global transformations of bounded domains. Trans. Am. Math. Soc. 368(4), 2679-2696 (2016)

2. Deng, F., Fornæss, J.E., Wold, E.F.: Exposing boundary points of strongly pseudoconvex subvarieties in complex spaces. Proc. Am. Math. Soc. 146(6), 2473-2487 (2018)

3. Diederich, K., Fornæss, J.E., Wold, E.F.: Exposing points on the boundary of strictly pseudoconvex or a locally convexifiable domain of finite 1-type. J. Geom. Anal. 24, 2124-2134 (2014)

4. Forstnerič, F.: Noncritical holomorphic functions on Stein manifolds. Acta Math. 191(2), 143-189 (2003)

5. Forstnerič, F., Wold, E.F.: Bordered Riemann surfaces in $\mathbb{C}^{2}$. J. Math. Pures Appl. (9) 91(1), 100-114 (2009)

6. Forstnerič, F.: Stein Manifolds and Holomorphic Mappings. The Homotopy Principle in Complex Analysis, 2nd edn. Ergebnisse der Mathematik und ihrer Grenzgebiete. 3. Folge/A Series of Modern Surveys in Mathematics 56. Springer, Berlin (2017)

7. Glaeser, G.: Étude de quelques algèbres tayloriennes. J. Anal. Math. 6, 1-124 (1958); Erratum, insert to $6(2)(1958)$

8. Gong, X., Kim, K.-T.: The $\bar{\partial}$-equation on variable strictly pseudoconvex domains. Math. Z. 290(1-2), 111-144 (2018)

9. Hirsch, M.W.: Differential Topology. Graduate Texts in Mathematics, No. 33, Springer, New York (1976)

10. Hörmander, L.: Generators of some rings of analytic functions. Bull. AMS 73, 943-949 (1967)

11. Krantz, S.G.: Function Theory of Several Complex Variables. AMS Chelsea Publishing, Providence (2001) (Reprint of the 1992 Edition)

12. Krantz, S.G.: The corona problem in several complex variables. In: Douglas, R.G., Krantz, S.G., Sawyer, E.T., Treil, S., Wick, B.D. (eds.) The Corona Problem. Connections Between Operator Theory, Function Theory, and Geometry. Fields Institute Communications, vol. 72, pp. 107-126. Springer, Berlin (2014)

13. Lewandowski, A.: Families of exposing maps in strictly pseudoconvex domains. J. Geom. Anal. (2019). https://doi.org/10.1007/s12220-019-00294-0

14. Lieb, I., Michel, J.: The Cauchy-Riemann Complex. Integral Formulae And Neumann Problem. Aspects of Mathematics, E34. Friedr. Vieweg \& Sohn, Braunschweig (2002)

15. Lieb, I., Range, R.M.: Lösungsoperatoren für den Cauchy-Riemann-Komplex mit $\mathcal{C}^{k}$-Abschätzungen. Math. Ann. 253(2), 145-164 (1980)

16. Malgrange, B.: Ideals of Differentiable Functions. Tata Institute of Fundamental Research Studies in Mathematics, No. 3. Tata Institute of Fundamental Research/Oxford University Press, Bombay/London (1966)

17. Range, R.M.: Holomorphic Functions and Integral Representations in Several Complex Variables. Graduate Texts in Mathematics, vol. 108. Springer (1986) (Corrected second printing, 1998)

18. Simon, L.: A parameter version of Forstnerič's splitting lemma. J. Geom. Anal. 29, 2124-2146 (2019)

19. Whitney, H.: Analytic extensions of differentiable functions defined in closed sets. Trans. Am. Math. Soc. 36(1), 63-89 (1934)

Publisher's Note Springer Nature remains neutral with regard to jurisdictional claims in published maps and institutional affiliations. 